\title{
Discrimination of hospital isolates of Acinetobacter baumannii using repeated sequences and whole genome alignment differential analysis
}

\author{
Roman Kotłowski $^{1}$ (D) Alicja Nowak-Zaleska ${ }^{2}$ - Grzegorz Węgrzyn²
}

Received: 22 December 2020 / Revised: 8 March 2021 / Accepted: 19 May 2021 / Published online: 8 June 2021

(c) The Author(s) 2021

\begin{abstract}
An optimized method for bacterial strain differentiation, based on combination of Repeated Sequences and Whole Genome Alignment Differential Analysis (RS\&WGADA), is presented in this report. In this analysis, 51 Acinetobacter baumannii multidrug-resistance strains from one hospital environment and patients from 14 hospital wards were classified on the basis of polymorphisms of repeated sequences located in CRISPR region, variation in the gene encoding the EmrA-homologue of $E$. coli, and antibiotic resistance patterns, in combination with three newly identified polymorphic regions in the genomes of A. baumannii clinical isolates. Differential analysis of two similarity matrices between different genotypes and resistance patterns allowed to distinguish three significant correlations $(p<0.05)$ between 172 bp DNA insertion combined with resistance to chloramphenicol and gentamycin. Interestingly, 45 and 55 bp DNA insertions within the CRISPR region were identified, and combined during analyses with resistance/susceptibility to trimethoprim/sulfamethoxazole. Moreover, 184 or 1374 bp DNA length polymorphisms in the genomic region located upstream of the GTP cyclohydrolase I gene, associated mainly with imipenem susceptibility, was identified. In addition, considerable nucleotide polymorphism of the gene encoding the gamma/tau subunit of DNA polymerase III, an enzyme crucial for bacterial DNA replication, was discovered. The differentiation analysis performed using the above described approach allowed us to monitor the distribution of A. baumannii isolates in different wards of the hospital in the time frame of several years, indicating that the optimized method may be useful in hospital epidemiological studies, particularly in identification of the source of primary infections.
\end{abstract}

Keywords Acinetobacter baumannii · Hospital infections · DNA polymerase III gene DNA polymerase III subunit gamma/ tau $\cdot$ Genetic polymorphisms $\cdot$ Antibiotics $\cdot$ Assembled matrix data

Communicated by Agnieszka Szalewska-Palasz.

Alicja Nowak-Zaleska

alicja.nowak-zaleska@ug.edu.pl; ala.kol.zal@gmail.com

Roman Kotłowski

romkotlo@pg.edu.pl

Grzegorz Węgrzyn

Grzegorz.wegrzyn@ug.edu.pl

1 Department of Molecular Biotechnology and Microbiology, Faculty of Chemistry, Gdansk University of Technology, Gabriela Narutowicza 11/12 street, 80-233 Gdansk, Poland

2 Department of Molecular Biology, Faculty of Biology, Gdansk University, Wita Stwosza 59 street, 80-308 Gdańsk, Poland

\section{Introduction}

The genome of each microorganism is a source of knowledge that can be applied for strain differentiation, based on bioinformatic tools and available techniques of molecular biology, suitable for epidemiological investigations. Among the species of the genus Acinetobacter, A. baumannii strains manifest the highest pathogenicity (Wong et al. 2017; Skariyachan et al. 2019). They are highly opportunistic microorganisms, responsible for hospital infections related to ability to adapt to different environmental conditions (Antunes et al. 2014). At the beginning of the twenty-first century, no complete genome sequence of Acinetobacter sp. was known. Barbe et al. (2004) published the first sequence of Acinetobacter sp. ADP1 genome, and later Smith et al. (2007) published the complete genome of A. baumannii ATCC 17,978 . Subsequently, the first genomic sequence of the 
multidrug-resistant $A$. baumannii strain was published by Adams et al. (2008). Presently, complete sequences of the genomic DNA of A. baumannii are known for about 250 strains (http://www.ncbi.nlm.nih.gov, database retrieved on 10 December 2020). As indicated by various research teams, repeated sequences of $A$. baumannii and other microorganisms have great impact in the process of generating pathogenicity for immunocompromised hosts (Zhou et al. 2014; Shariat and Dudley 2014; Nabil et al. 2015) or adaptation skills to different environmental conditions (Zhou et al. 2014; Shariat and Dudley 2014; Karah et al. 2015).

The presence of tandem DNA repeats in genomes of $A$. baumannii was confirmed by several groups (Martín-Lozano et al. 2002; Turton et al. 2009; Irfan et al. 2011; Pourcel et al. 2011; Minandri et al. 2012; Ergin et al. 2013; Ahmed and Alp 2015; Villalón et al. 2015). Based on these sequences, different methods of differentiation of A. baumannii strains have been developed; however, they take into account only their diversifying power of evolutionary changes of the Acinetobacter genus (Touchon et al. 2014). Thus, their features responsible for drug resistance or pseudo-immunological bacterial responses, encoded in the Clustered Regularly Interspaced Short Palindromic Repeats (CRISPR) system, which evolved to protect the cells from exogenous phage and plasmid DNA invasion, are ignored in such analyses. On the other hand, as suggested by Touchon et al. (2014), the next step in the process of strains' classification should be focused on confrontation of the genetic and phenotypic features related to pathogenicity of bacterial species. To address some of the above features, an optimized method for A. baumannii differential analysis is proposed in this report. It is based on combination of the previously described method based on analysis of repeated sequences (Nowak-Zaleska et al. 2008, 2016) and whole genome alignment.

\section{Materials and methods}

\section{Bacterial strains}

We used 51 A. baumannii isolates from diagnostic materials of the hospital environments of Antoni Jurasz University Hospital in Bydgoszcz. These isolates were derived from 11 hospital wards (Dermatology, Endocrinology, Geriatrics, General and Endocrine Surgery, General and Vascular Surgery, Intensive Care Units, Neurology, Nephrology, Neurosurgery, Orthopedic, Plastic Surgery), 2 clinics (Orthopedic Outpatient Clinic, Surgical Outpatient Clinic), and Rehabilitation Department. The isolates were collected during the period of 2003-2006 (Table 1). The following strains were isolated from different diagnostic materials: 10 from bronchoalveolar lavages, 8 from bedsores, 2 from blood, 1 from cerebrospinal fluid, 2 from drains, 2 from drain swabs,
1 from needle tip, 1 from pus, 8 from respiratory secretions, 2 from tracheostomy tube swabs, 1 from tube swab, 9 from ulceration wounds, and 4 from urine. Strains were identified based on ID GN phenotypic identification system, including drug sensitivity. This identification was conducted using Kirby-Bauer method, according to CLSI instructions (for details, see Nowak-Zaleska et al. 2008, 2016).

\section{Locus identification with repeated sequences}

The isolates of A. baumannii were differentiated on the basis of previously published polymorphisms of repeated sequences located in the CRISPR region (Touchon et al. 2014), variation in the gene encoding the EmrA homologue of $E$. coli (Nowak-Zaleska et al. 2016), and three newly identified (in this study) polymorphic regions (Tables 2 and 3).

\section{DNA-technology methods}

The genetic material from the isolates was obtained using Genomic Mini Set, purchased from A\&A Biotechnology (Gdynia, Poland), following the manufacturer's instruction. For the DR-PCR/RFLP genotyping method, sequences of primers, the PCR reaction conditions, and enzymatic digestion of PCR products were previously described (NowakZaleska et al. 2008). Briefly, the amplification reactions were conducted according to the following time-temperature profile: $94{ }^{\circ} \mathrm{C}$ for $2 \mathrm{~min}$, during the initial denaturation step, 35 cycles consisting of the DNA denaturation at $94{ }^{\circ} \mathrm{C}$ for $1 \mathrm{~min}$, hybridization at $68{ }^{\circ} \mathrm{C}$ for $1 \mathrm{~min}$, and extension at $72{ }^{\circ} \mathrm{C}$ for $2 \mathrm{~min}$. The amplification products were subjected to the restriction fragment length polymorphism (RFLP) analysis using HaeIII and SsiI restriction enzymes. Separation of restriction fragments was performed electrophoretically, in $12 \%$ polyacrylamide gels, and results were documented using Versa Doc Imaging System, ver. 1000. The homologous region of the emrA resistance-related gene, containing 6-nt repeats, was analyzed as described previously (Nowak-Zaleska et al. 2016). Identification of three newly discovered polymorphic regions was possible after multiple alignment of nine A. baumannii genomes (see Table 2), using the MAFFT 7.271 software (Katoh et al. 2002). Subsequently, three pairs of primers, shown in Table 3, were used in the PCR analysis. The PCR reactions were conducted in $25 \mu 1$ reaction mixtures, using the Eppendorf AG 22,331 thermal cycler. The PCR mixtures were as follows: $1.5 \mathrm{U}$ of RUN DNA polymerase (purchased from A\&A Biotechnology), PCR reaction buffer containing $10 \mathrm{mM} \mathrm{KCl}, 10 \mathrm{mM}$ $\left(\mathrm{NH}_{4}\right)_{2} \mathrm{SO}_{4}, 0.1 \%$ Triton X-100, $20 \mathrm{mM}$ Tris, $\mathrm{pH} 8.5,2 \mathrm{mM}$ of $\mathrm{Mg}_{2} \mathrm{Cl}, 2 \mathrm{mM}$ of each deoxynucleoside triphosphates, $25 \mathrm{pM}$ of suitable pairs of primers, and $50 \mathrm{ng} / \mu \mathrm{l}$ of template DNA. Amplified PCR products were separated using $2 \%$ agarose gel electrophoresis and standard ethidium bromide 
Table 1 Characteristics of multidrug-resistant Acinetobacter baumannii clinical isolates

\begin{tabular}{|c|c|c|c|c|c|}
\hline No & Isolates $^{*}$ & Antibiogram $^{a}$ & Genotype pattern $^{\mathrm{b}}$ & $\begin{array}{l}\text { Combined analysis } \\
\text { cluster }^{c}\end{array}$ & Source of isolates \\
\hline 1 & 2005VI.70.ICU & I & 1 & 1 & Ulceration wound \\
\hline 2 & 2006III.107.NS & II & 8 & 2 & Respiratory secretion \\
\hline 3 & 2006I.96.ICU & II & 8 & 2 & BAL \\
\hline 4 & 2006I.95.ICU & II & 8 & 2 & BAL \\
\hline 5 & 2006I.93.R & II & 8 & 2 & Urine \\
\hline 6 & 2006I.92.ICU & II & 8 & 2 & BAL \\
\hline 7 & 2006II.105.E & II & 7 & 3 & Respiratory secretion \\
\hline 8 & 2006IV.108.NS & II & 7 & 3 & $\mathrm{CSF}$ \\
\hline 9 & 2005XI.85.ICU & II & 8 & 2 & BAL \\
\hline 10 & 2005XII.91.ICU & II & 8 & 2 & BAL \\
\hline 11 & 2005XI.88.R & II & 8 & 2 & Urine \\
\hline 12 & 2005XI.87.PS & II & 8 & 2 & Bedsores \\
\hline 13 & 2006II.98.R & II & 8 & 2 & Respiratory secretion \\
\hline 14 & 2005VI.71.R & II & 10 & 4 & Respiratory secretion \\
\hline 15 & 2006II.100.G & II & 10 & 4 & Urine \\
\hline 16 & 2006II.101.ICU & II & 10 & 4 & Blood \\
\hline 17 & 2005X.79.NS & II & 10 & 4 & Urine \\
\hline 18 & 2006II.102.ICU & II & 9 & 5 & BAL \\
\hline 19 & 2005IV.68.R & II & 13 & 6 & Drain swab \\
\hline 20 & 2003VI.43.G\&ES & II & 6 & 7 & Ulceration wound \\
\hline 21 & 2003VIII.45.O & II & 6 & 7 & Drain swab \\
\hline 22 & 2003IX.48.N & II & 6 & 7 & Tracheostomy tube swab \\
\hline 23 & 2004XI.61.O & II & 15 & 8 & Ulceration wound \\
\hline 24 & 2004X.59.OC & III & 4 & 9 & Bedsores \\
\hline 25 & 2006I.94.NS & IV & 8 & 10 & Respiratory secretion \\
\hline 26 & 2006II.104.NS & IV & 7 & 11 & Respiratory secretion \\
\hline 27 & 2004VIII.55.OC & V & 2 & 12 & Bedsores \\
\hline 28 & 2003XI.50.O & $\mathrm{V}$ & 6 & 13 & Bedsores \\
\hline 29 & 2005I.65.O & V & 4 & 14 & Drain \\
\hline 30 & 2003IX.47.ICU & VI & 6 & 15 & BAL \\
\hline 31 & 2005VIII.72.G\&ES & VI & 15 & 16 & Ulceration wound \\
\hline 32 & 2003VIII.44.ICU & VII & 6 & 17 & Ulceration wound \\
\hline 33 & 2003IX.46.G\&ES & VII & 6 & 17 & Ulceration wound \\
\hline 34 & 2003III.42.ICU & VII & 12 & 18 & Tracheostomy tube swab \\
\hline 35 & 2003IX.49.D & VIII & 14 & 19 & Ulceration wound \\
\hline 36 & 2005IV.67.ICU & IX & 15 & 20 & Ulceration wound \\
\hline 37 & 2004IV.52.E & $X$ & 15 & 21 & Bedsores \\
\hline 38 & 2006II.103.ICU & $X$ & 15 & 21 & BAL \\
\hline 39 & 2004X.58.R & $X$ & 15 & 21 & Tube swab \\
\hline 40 & 2005III.66.O & XI & 4 & 22 & Drain \\
\hline 41 & 2004X.56.NS & XII & 11 & 23 & Blood \\
\hline 42 & 2004X.57.NS & XII & 15 & 24 & Bedsores \\
\hline 43 & 2004XI.63.R & XIII & 3 & 25 & Pus \\
\hline 44 & 2004VIII.54.ICU & XIII & 5 & 26 & BAL \\
\hline 45 & 2004XI.62.G & XIII & 4 & 27 & Bedsores \\
\hline 46 & 2005 V.69.SC & XIII & 15 & 28 & Ulceration wound \\
\hline 47 & 2004VI.53.N & XIV & 16 & 29 & Bedsores \\
\hline 48 & 2006II.106.NS & $X V$ & 8 & 30 & Respiratory secretion \\
\hline 49 & 2005XII.90.Nef & $\mathrm{XV}$ & 8 & 30 & Respiratory secretion \\
\hline
\end{tabular}


Table 1 (continued)

\begin{tabular}{|c|c|c|c|c|c|}
\hline No & Isolates* & Antibiogram $^{\mathrm{a}}$ & Genotype pattern ${ }^{b}$ & $\begin{array}{l}\text { Combined analysis } \\
\text { cluster }^{c}\end{array}$ & Source of isolates \\
\hline 50 & 2005IX.76.ICU & $\mathrm{XV}$ & 10 & 31 & BAL \\
\hline 51 & 2005IX.78.G\&VS & $\mathrm{XV}$ & 10 & 31 & Needle tip \\
\hline HGDI index & & 0.8 & 0.8816 & 0.9718 & \\
\hline
\end{tabular}

${ }^{a}$ For details of particular antibiogram patterns, see Table 5

${ }^{\mathrm{b}}$ For details of particular genotype patterns, see Table 4

${ }^{c}$ Numbers arisen from combination of antibiogram and genotype patterns

*Abbreviations for isolates (the last letter(s) in the name): D—Dermatology, E-Endocrinology, G-Geriatrics, G\&ES-General and Endocrine Surgery, G\&VS—General and Vascular Surgery, ICU—Intensive Care Unit, N—Neurology, Nef-Nephrology, NS—Neurosurgery, O-Orthopedic, OC-Orthopedic Outpatient Clinic, PS-Plastic Surgery, R-Rehabilitation, SC-Surgical Outpatient Clinic

\#Abbreviations for source of isolates: BAL—bronchoalveolar lavage; CSF-cerebrospinal fluid

staining procedure (Sambrook et al. 1989). Images of the gels were obtained using Versa Doc Imaging System, ver. 1000 .

\section{Statistical analysis}

Statistical analysis was performed using Epi Info 7.2.3.1 software using two-tailed Fisher exact test analysis. The values " 1 " and " 0 " were representing resistant and susceptible strains for different antibiotics used in our study. Similarity matrices of different genotypes and resistance features and phylogenetic trees were constructed using package MVSP ver. 3.22 .

\section{Results and discussion}

To enhance the currently available methods of differentiation of A. baumannii strains, we were searching for previously unknown PCR-derived fragment length polymorphism variations in randomly identified regions of selected genomic sequences. The theoretical values of PCR fragment lengths of the newly discovered polymorphic regions for nine $A$. baumannii genomes are presented in Table 2. Among three identified polymorphic regions, only one was characterized by the highest length polymorphism. It was recognized as a gene fragment coding for DNA polymerase III subunit gamma/tau, with the Protein_id=AFI95102.1 in the
Table 2 The sizes of PCR products for designed pairs of primers calculated for selected Acinetobacter baumannii genomes

\begin{tabular}{|c|c|c|c|}
\hline \multirow{3}{*}{$\begin{array}{l}\text { Genome NCBI accession numbers* of } \\
\text { Acinetobacter baumannii strains }\end{array}$} & \multicolumn{3}{|c|}{ PCR product length (bp) } \\
\hline & Genomic region 1 & Genomic region 2 & Genomic region 3 \\
\hline & $\begin{array}{l}\text { Primer pairs: } \\
\text { Aci7 and Aci8 }\end{array}$ & $\begin{array}{l}\text { Primer pairs: } \\
\text { Aci13 and Aci14 }\end{array}$ & $\begin{array}{l}\text { Primer pairs: } \\
\text { Aci17 and Aci18 }\end{array}$ \\
\hline $\begin{array}{l}\text { CP001172.2 } \\
\text { Acinetobacter baumannii AB307-0294 }\end{array}$ & 204 & 184 & 404 \\
\hline $\begin{array}{l}\text { NC_011586.2 } \\
\text { Acinetobacter baumannii AB0057 }\end{array}$ & 162 & 184 & 405 \\
\hline $\begin{array}{l}\text { CP002522. } 2 \\
\text { Acinetobacter baumannii TCDC-AB } 0715\end{array}$ & 180 & 236 & 508 \\
\hline $\begin{array}{l}\text { NC_010611.1 } \\
\text { Acinetobacter baumannii ACICU }\end{array}$ & 144 & 1274 & 508 \\
\hline $\begin{array}{l}\text { CP001937.2 } \\
\text { Acinetobacter baumannii MDR-ZJ06 }\end{array}$ & 222 & 1374 & 500 \\
\hline $\begin{array}{l}\text { CP003500.1 } \\
\text { Acinetobacter baumannii MDR-TJ }\end{array}$ & 222 & 1374 & 508 \\
\hline $\begin{array}{l}\text { CP003847.1 } \\
\text { Acinetobacter baumannii BJAB } 0715\end{array}$ & 156 & 186 & 406 \\
\hline $\begin{array}{l}\text { NZ_CP018664.1 } \\
\text { Acinetobacter baumannii ATCC } 17,978\end{array}$ & 210 & 185 & 306 \\
\hline $\begin{array}{l}\text { NC_010410.1 } \\
\text { Acinetobacter baumannii AYE }\end{array}$ & 234 & 1373 & 405 \\
\hline
\end{tabular}

*NCBI-National Center for Biotechnology Information 


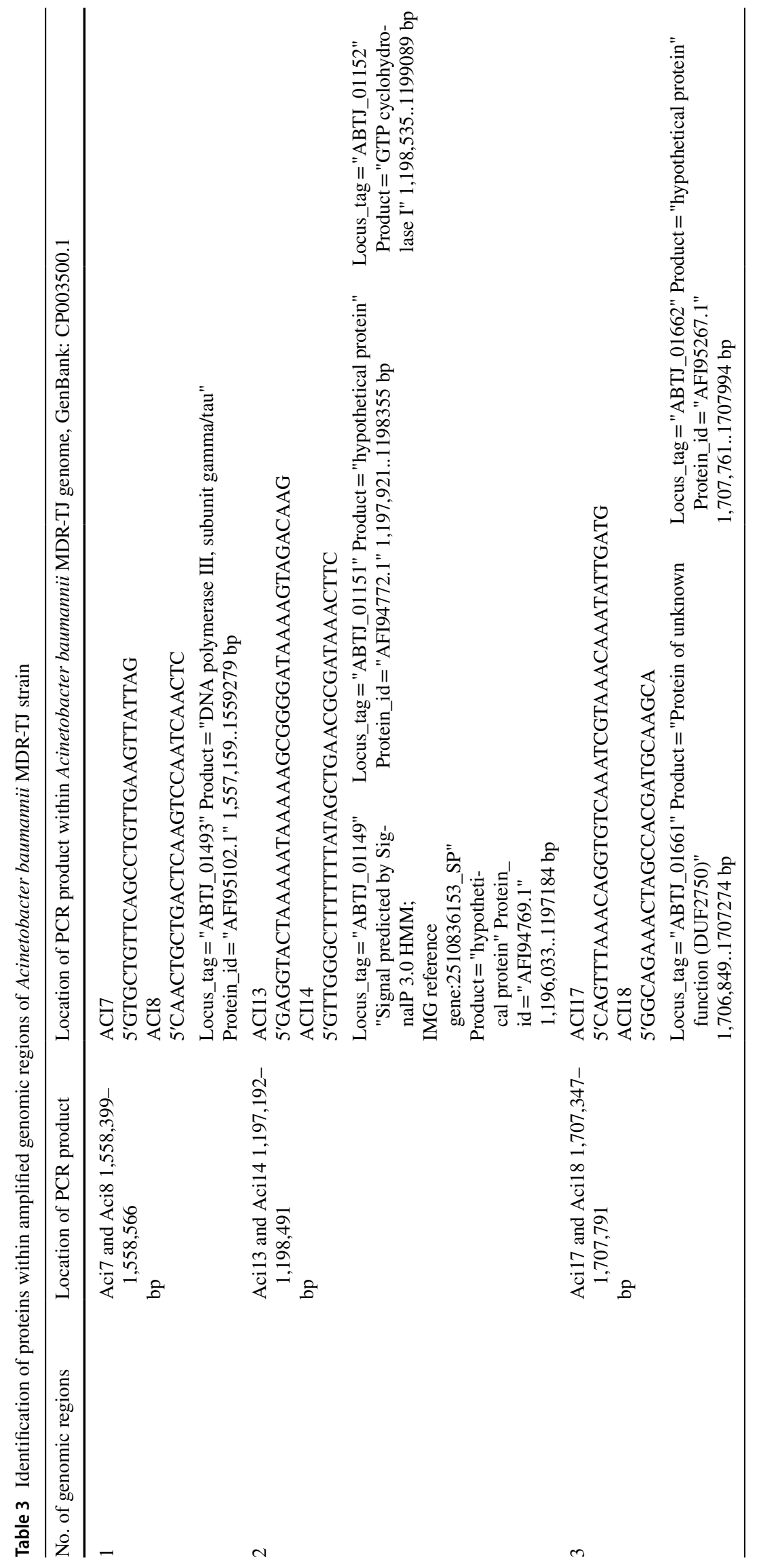


MDR-TJ A. baumannii genome (GenBank accession no. CP003500.1) (Table 3).

In order to increase variation of analyzed A. baumannii isolates, two other previously described variable regions in the genomes of A. baumannii were included in our study (Nowak-Zaleska et al. 2008, 2016). The combined application of the three genetic aforementioned genotypic methods, DR-PCR/RFLP, different number of P-A dipeptide repeats encoded in the N-terminal part of EmrA-homologue gene, as well as three new variables, namely, Aci7 and Aci8, Aci13 and Aci14, and Aci17 and Aci18 (Table 4), combined with known information about resistance patterns for each isolate (Table 5), allowed for recognition of 31 different clusters shown in Table 1.

Detailed analysis of bacterial isolates and diagnostic material revealed significant differences between A. baumannii isolates from bronchoalveolar lavage (BAL) and other clinical samples $(p<0.0001)$, as well as significant correlation between resistance pattern II and genotype $8^{\text {th }}$ $(p<0.01)$, presented in Table 1. In addition, significant correlation $(p<0.05)$ between the frequency of occurrence of $8^{\text {th }} A$. baumannii genotype in the first trimester of 2006 year in comparison to other periods of isolation time was also evident. Higher Hunter-Gaston Discriminatory Index (HGDI), presented in Table 1, was determined using the method developed in this study, in comparison to previously published genotyping methods (Nowak-Zaleska et al. 2008, 2016). Furthermore, in the course of statistical data analysis, we observed that strains representing clusters 2 and 4 from combined genetic-phenotypic analysis, shown in Table 1, were isolated in two consecutive years 2005 and $2006(p<0.0001)$. These strains represent the $15^{\text {th }}$ genotype pattern, which was present in 2004 and 2005, but with different resistance patterns II, VI, IX, X, XII, and XIII $(p=0.01)$. In addition, three strains representing $21^{\text {st }}$ cluster with the resistance pattern X appeared in years 2004 and 2006. Moreover, strains with genotypes 6,12 , and 14 were only present in $2003(p<0.0001)$, in comparison to other genotypes, and what is interesting, the resistance pattern II appeared each year, while patterns V, VI, VII, and VIII appeared only between 2003 and $2005(p=0.01)$.

Combined analysis of similarity matrices, obtained using data from Tables 4 and 5, revealed that out of 19 combinations of genetic and resistance markers, only three were significantly different $(p<0.05)$ (Table 6$)$, as indicated by $\chi^{2}$ value higher than 4 , obtained from two phylogenetic trees presented in Fig. 1. Among significantly different mixed parameters identified, there were (1) 172 bp DNA insertion, located in the CRISPR locus, identified using the $S s i$ I enzyme for genotypes 7 to 11 , in combination

Table 4 Set of different genotypes shown as PCR length polymorphisms in nucleotide base pairs for 51 MDR Acinetobacter baumannii isolates

\begin{tabular}{|c|c|c|c|c|c|c|c|c|c|c|c|c|c|c|c|c|c|c|c|}
\hline \multirow[t]{3}{*}{ Genotypes } & \multicolumn{3}{|c|}{$\begin{array}{l}\text { Three new PCR regions (length } \\
\text { in bp) }\end{array}$} & \multicolumn{15}{|c|}{ PCR-DR/RFLP region (length in bp) } & \multirow{3}{*}{$\begin{array}{l}\text { EmrA*-homo- } \\
\text { logue gene frag- } \\
\text { ment } \\
\text { (length in bp) }\end{array}$} \\
\hline & \multirow{2}{*}{$\begin{array}{l}\text { Genomic } \\
\text { region } 1 \\
\text { Aci7 and } \\
\text { Aci8 }\end{array}$} & \multirow{2}{*}{$\begin{array}{l}\text { Genomic } \\
\text { region } 2 \\
\text { Aci13 } \\
\text { and } \\
\text { Aci14 }\end{array}$} & \multirow{2}{*}{$\begin{array}{l}\text { Genomic } \\
\text { region } 3 \\
\text { Aci17 } \\
\text { and } \\
\text { Aci18 }\end{array}$} & \multicolumn{7}{|c|}{ HaeIII pattern } & \multicolumn{8}{|c|}{ SsiI pattern } & \\
\hline & & & & $\# 1$ & $\# 2$ & $\# 3$ & \#4 & $\# 5$ & $\# 6$ & $\# 7$ & $\# 1$ & $\# 2$ & \#3 & $\# 4$ & 5 & \#6 & $\# 7$ & $\# 8$ & \\
\hline 1 & 156 & 184 & 600 & 106 & 0 & 63 & 60 & 57 & 54 & 45 & 0 & 137 & 109 & 88 & 76 & 63 & 43 & 38 & 138 \\
\hline 2 & 234 & 184 & 405 & 107 & 83 & 78 & 64 & 60 & 59 & 55 & 0 & 0 & 111 & 0 & 74 & 61 & 43 & 38 & 126 \\
\hline 3 & 204 & 184 & 405 & 106 & 82 & 63 & 60 & 57 & 54 & 45 & 0 & 137 & 109 & 88 & 76 & 63 & 43 & 38 & 126 \\
\hline 4 & 210 & 184 & 405 & 106 & 82 & 63 & 60 & 57 & 54 & 45 & 0 & 137 & 109 & 88 & 76 & 63 & 43 & 38 & 126 \\
\hline 5 & 234 & 184 & 405 & 106 & 82 & 63 & 60 & 57 & 54 & 45 & 0 & 137 & 109 & 88 & 76 & 63 & 43 & 38 & 126 \\
\hline 6 & 222 & 184 & 405 & 106 & 82 & 63 & 60 & 57 & 54 & 45 & 0 & 137 & 109 & 88 & 76 & 63 & 43 & 38 & 126 \\
\hline 7 & 234 & 1374 & 508 & 106 & 82 & 63 & 60 & 57 & 54 & 45 & 172 & 134 & 110 & 89 & 76 & 63 & 42 & 37 & 126 \\
\hline 8 & 222 & 1374 & 508 & 106 & 82 & 63 & 60 & 57 & 54 & 45 & 172 & 134 & 110 & 89 & 76 & 63 & 42 & 37 & 126 \\
\hline 9 & 210 & 1374 & 508 & 106 & 82 & 63 & 60 & 57 & 54 & 45 & 172 & 134 & 110 & 89 & 76 & 63 & 42 & 37 & 132 \\
\hline 10 & 210 & 1374 & 508 & 106 & 82 & 63 & 60 & 57 & 54 & 45 & 172 & 134 & 110 & 89 & 76 & 63 & 42 & 37 & 126 \\
\hline 11 & 180 & 1374 & 508 & 106 & 82 & 63 & 60 & 57 & 54 & 45 & 172 & 134 & 110 & 89 & 76 & 63 & 42 & 37 & 120 \\
\hline 12 & 144 & 1374 & 306 & 109 & 77 & 71 & 64 & 58 & 55 & 0 & 0 & 137 & 109 & 88 & 76 & 63 & 43 & 38 & 132 \\
\hline 13 & 210 & 1374 & 405 & 106 & 82 & 63 & 60 & 57 & 54 & 45 & 0 & 137 & 109 & 88 & 76 & 63 & 43 & 38 & 126 \\
\hline 14 & 210 & 1374 & 405 & 106 & 82 & 63 & 60 & 57 & 54 & 45 & 0 & 137 & 109 & 88 & 76 & 63 & 43 & 38 & 132 \\
\hline 15 & 156 & 1374 & 306 & 109 & 77 & 71 & 64 & 58 & 55 & 0 & 0 & 137 & 109 & 88 & 76 & 63 & 43 & 38 & 132 \\
\hline 16 & 162 & 1374 & 306 & 109 & 77 & 71 & 64 & 58 & 55 & 0 & 0 & 137 & 109 & 88 & 76 & 63 & 43 & 38 & 132 \\
\hline
\end{tabular}

${ }^{*}$ EmrA — an enzyme from Escherichia coli

\#_restriction pattern number 
Table 5 Set of different antibiotic resistance patterns determined for 51 MDR Acinetobacter baumannii strains

\begin{tabular}{|c|c|c|c|c|c|c|c|c|c|c|c|c|c|}
\hline \multirow{2}{*}{$\begin{array}{l}\text { Resist- } \\
\text { ance } \\
\text { pattern }\end{array}$} & \multicolumn{13}{|c|}{ Antibiotic resistance/susceptibility } \\
\hline & IPM & NET & $\mathrm{NN}$ & CAZ & CIP & CTX & CFP & TIC & ATM & SXT & $\mathrm{C} / \mathrm{GM}$ & $\mathrm{GM} / \mathrm{C}$ & AN \\
\hline I & $\mathrm{R}$ & $\mathrm{S}$ & $\mathrm{R}$ & $\mathrm{S}$ & $\mathrm{R}$ & $\mathrm{R}$ & $\mathrm{R}$ & $\mathrm{R}$ & $\mathrm{R}$ & $\mathrm{R}$ & $\mathrm{R}$ & $\mathrm{R}$ & $\mathrm{R}$ \\
\hline II & $\mathrm{S}$ & $\mathrm{R}$ & $\mathrm{R}$ & $\mathrm{R}$ & $\mathrm{R}$ & $\mathrm{R}$ & $\mathrm{R}$ & $\mathrm{R}$ & $\mathrm{R}$ & $\mathrm{R}$ & $\mathrm{R}$ & $\mathrm{R}$ & $\mathrm{R}$ \\
\hline III & $S$ & $\mathrm{R}$ & $S$ & $\mathrm{R}$ & $\mathrm{R}$ & $\mathrm{R}$ & $\mathrm{R}$ & $\mathrm{R}$ & $\mathrm{R}$ & $\mathrm{R}$ & $\mathrm{R}$ & $\mathrm{R}$ & $\mathrm{R}$ \\
\hline IV & $\mathrm{S}$ & $\mathrm{R}$ & $\mathrm{R}$ & $\mathrm{R}$ & $\mathrm{R}$ & $\mathrm{R}$ & $\mathrm{R}$ & $\mathrm{R}$ & $\mathrm{R}$ & $\mathrm{R}$ & $\mathrm{R}$ & $\mathrm{R}$ & $\mathrm{S}$ \\
\hline V & $\mathrm{S}$ & $\mathrm{R}$ & $\mathrm{R}$ & $\mathrm{R}$ & $\mathrm{S}$ & $\mathrm{R}$ & $\mathrm{R}$ & $\mathrm{R}$ & $\mathrm{R}$ & $\mathrm{R}$ & $\mathrm{R}$ & $\mathrm{R}$ & $\mathrm{R}$ \\
\hline VI & $S$ & $\mathrm{~S}$ & $\mathrm{R}$ & $\mathrm{R}$ & $\mathrm{R}$ & $\mathrm{R}$ & $\mathrm{R}$ & $\mathrm{R}$ & $\mathrm{R}$ & $\mathrm{R}$ & $\mathrm{R}$ & $\mathrm{R}$ & $\mathrm{R}$ \\
\hline VII & $S$ & $\mathrm{~S}$ & $S$ & $\mathrm{R}$ & $\mathrm{R}$ & $\mathrm{R}$ & $\mathrm{R}$ & $\mathrm{R}$ & $\mathrm{R}$ & $\mathrm{R}$ & $\mathrm{R}$ & $\mathrm{R}$ & $\mathrm{R}$ \\
\hline VIII & $S$ & $S$ & $S$ & $S$ & $S$ & $S$ & $\mathrm{~S}$ & $S$ & $\mathrm{~S}$ & $\mathrm{~S}$ & $\mathrm{R}$ & $\mathrm{R}$ & $\mathrm{R}$ \\
\hline IX & $S$ & $S$ & $\mathrm{R}$ & $\mathrm{R}$ & $\mathrm{R}$ & $\mathrm{R}$ & $\mathrm{R}$ & I & $\mathrm{R}$ & $\mathrm{R}$ & $\mathrm{R}$ & $\mathrm{R}$ & $\mathrm{R}$ \\
\hline$X$ & $\mathrm{~S}$ & $S$ & I & $\mathrm{R}$ & $\mathrm{R}$ & $\mathrm{R}$ & $\mathrm{R}$ & I & $\mathrm{R}$ & $\mathrm{R}$ & $\mathrm{R}$ & $\mathrm{R}$ & $\mathrm{R}$ \\
\hline XI & $\mathrm{S}$ & $\mathrm{R}$ & $\mathrm{R}$ & $\mathrm{R}$ & I & $\mathrm{R}$ & $\mathrm{R}$ & $\mathrm{R}$ & I & $\mathrm{R}$ & $\mathrm{R}$ & $\mathrm{R}$ & $\mathrm{R}$ \\
\hline XII & $\mathrm{S}$ & I & $\mathrm{R}$ & $\mathrm{R}$ & $\mathrm{R}$ & $\mathrm{R}$ & $\mathrm{R}$ & $\mathrm{R}$ & $\mathrm{R}$ & $\mathrm{R}$ & $\mathrm{R}$ & $\mathrm{R}$ & $\mathrm{R}$ \\
\hline XIII & $\mathrm{S}$ & $\mathrm{R}$ & $\mathrm{R}$ & $\mathrm{R}$ & $\mathrm{R}$ & $\mathrm{R}$ & $\mathrm{R}$ & I & $\mathrm{R}$ & $\mathrm{R}$ & $\mathrm{R}$ & $\mathrm{R}$ & $\mathrm{R}$ \\
\hline XIV & $\mathrm{S}$ & $\mathrm{R}$ & I & $\mathrm{R}$ & $\mathrm{R}$ & $\mathrm{R}$ & $\mathrm{R}$ & I & $\mathrm{R}$ & $\mathrm{R}$ & $\mathrm{R}$ & $\mathrm{R}$ & $\mathrm{R}$ \\
\hline XV & $\mathrm{S}$ & $\mathrm{R}$ & $\mathrm{R}$ & $\mathrm{R}$ & $\mathrm{R}$ & $\mathrm{R}$ & $\mathrm{R}$ & $\mathrm{R}$ & $\mathrm{R}$ & $\mathrm{R}$ & $\mathrm{R}$ & $\mathrm{R}$ & I \\
\hline
\end{tabular}

Meaning of symbols: R, resistance; S, susceptibility; I, intermediate phenotype

Antibiotics abbreviations: AN, amikacin; ATM, aztreonam; C, chloramphenicol; CAZ, ceftazidime; CFP, cefoperazone; CIP, ciprofloxacin; CTX, cefotaxime; GM, gentamycin; IPM, imipenem; NN, tobramycin; NET, netilmicin; SXT, trimethoprim/sulfamethoxazole; TIC, ticarcillin

Identical results for GM and C for different restriction patterns SsiI_1 and SsiI_2 are named C/GM and $\mathrm{GM} / \mathrm{C}$
Table 6 Set of two joined-similarity matrices obtained for 19 different genotypes indicated by underlined values, and for 19 different antibiotic resistance patterns. All values are from the range between 1 and $100 \%$. Abbreviations "_s" and "_r" indicate intermediate resistance patterns considered two times as susceptible or resistant, respectively. The "0" value was replaced by "1E-06" for diagonal correlation calculation purposes. Significant $(p<0.05)$ combinations of genetic and resistance/susceptibility features are highlighted in black

\begin{tabular}{|c|c|c|c|c|c|c|c|c|c|c|c|c|c|c|c|c|c|c|c|}
\hline \multirow[b]{2}{*}{$\begin{array}{c}\text { Resistance } \\
\text { features }\end{array}$} & \multicolumn{19}{|c|}{ Genotypes } \\
\hline & 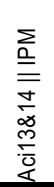 & 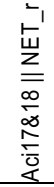 & $\begin{array}{l}z^{\prime} \\
z \\
\underline{z} \\
\infty \\
\infty \\
\frac{1}{0} \\
\frac{1}{4}\end{array}$ & 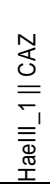 & 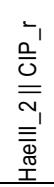 & 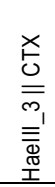 & 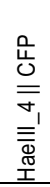 & 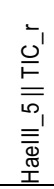 & 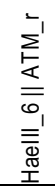 & 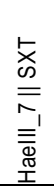 & $\begin{array}{l}\sum \\
\text { U } \\
= \\
\bar{E} \\
\bar{D}\end{array}$ & $\begin{array}{l}\mathcal{U} \\
\sum_{0} \\
\overline{\bar{N}} \\
\overline{\bar{N}} \\
\bar{\infty}\end{array}$ & $\begin{array}{l}\Sigma_{1} \\
z \\
\overline{\bar{m}} \\
\bar{m} \\
\bar{\infty}\end{array}$ & 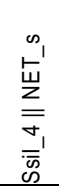 & $\begin{array}{l}\infty_{1} \\
z \\
z \\
\bar{n} \\
n_{1} \\
\bar{\infty} \\
\infty\end{array}$ & $\begin{array}{l}\infty_{1} \\
0^{\prime} \\
\overline{0} \\
\overline{0} \\
\bar{\infty} \\
\infty \\
\infty\end{array}$ & 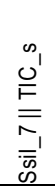 & $\begin{array}{l}\infty_{1} \\
z \\
\leq \\
\bar{\infty} \\
\bar{\infty} \\
\bar{\infty} \\
\infty\end{array}$ & 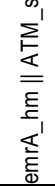 \\
\hline Aci13\&14 || IPM & 78.9 & $\underline{49.4}$ & 33.4 & $\underline{20.6}$ & $\underline{15.1}$ & 13.2 & $\underline{12.3}$ & $\underline{11.6}$ & 11.1 & $\underline{7.7}$ & 11.0 & 24.2 & 21.1 & 16.4 & 15.1 & 12.7 & $\underline{8.8}$ & $\underline{7.8}$ & 24.3 \\
\hline Aci17\&18 || NET_r & 0.0 & $\overline{65.2}$ & $\overline{63.4}$ & $\overline{39.7}$ & $\overline{30.0}$ & $\overline{26.4}$ & $\overline{24.8}$ & $\overline{23.5}$ & $\overline{22.5}$ & $\overline{15.9}$ & $\overline{22.2}$ & $\overline{45.7}$ & $\overline{\overline{40.5}}$ & $\overline{32.2}$ & $\overline{29.9}$ & $\overline{25.5}$ & $\overline{18.0}$ & $\overline{\overline{16.1}}$ & $\overline{45.9}$ \\
\hline Aci7\&8 \| NN_r & 15.4 & 76.2 & $\overline{40.6}$ & $\overline{69.6}$ & $\overline{\overline{55.1}}$ & $\overline{\underline{49.3}}$ & $\overline{46.8}$ & $\overline{\overline{44.6}}$ & $\overline{\underline{42.9}}$ & $\overline{\underline{31.4}}$ & $\overline{42.4}$ & $\overline{77.9}$ & $\overline{\overline{70.8}}$ & $\overline{\overline{58.6}}$ & $\overline{55.0}$ & $\overline{\overline{47.9}}$ & $\overline{\underline{35.2}}$ & $\overline{31.7}$ & $\overline{\overline{78.2}}$ \\
\hline HaellI_1 || CĀZ & 0.0 & 81.8 & 88.0 & $\overline{47.8}$ & $\overline{83.2}$ & $\overline{76.1}$ & $\overline{72.8}$ & $\overline{70.0}$ & $\overline{67.6}$ & $\overline{51.7}$ & $\overline{41.3}$ & $\overline{85.4}$ & $\overline{98.7}$ & $\overline{87.4}$ & $\overline{83.2}$ & $\overline{74.2}$ & $\overline{57.2}$ & $\overline{52.2}$ & $\overline{90.8}$ \\
\hline HaellI_2 \|CIP_r & 14.3 & 72.7 & 88.0 & 92.3 & $\overline{45.6}$ & $\overline{87.0}$ & $\overline{83.6}$ & $\overline{80.7}$ & $\overline{78.4}$ & $\overline{60.7}$ & $\overline{39.5}$ & $\overline{69.6}$ & $\overline{82.0}$ & $\overline{89.2}$ & $\overline{93.7}$ & $\overline{84.9}$ & $\overline{67.4}$ & $\overline{\underline{62.1}}$ & $\overline{74.4}$ \\
\hline HaellI_3 || CTX & 13.3 & 78.3 & 92.3 & 96.3 & 96.3 & $\overline{40.3}$ & $\overline{96.5}$ & $\overline{93.4}$ & $\overline{90.9}$ & $\overline{72.5}$ & 33.0 & $\overline{62.8}$ & $\overline{74.8}$ & $\overline{81.7}$ & $\overline{92.3}$ & $\overline{98.0}$ & $\overline{79.0}$ & $\overline{73.1}$ & $\overline{67.6}$ \\
\hline HaellI_4 || CFP & 13.3 & 78.3 & 92.3 & 96.3 & 96.3 & 100 & $\overline{38.9}$ & $\overline{\overline{96.9}}$ & $\overline{\overline{94.4}}$ & $\overline{75.7}$ & $\overline{32.7}$ & $\overline{60.5}$ & $\overline{\overline{71.6}}$ & $\overline{79.3}$ & $\overline{89.1}$ & $\overline{\overline{97.9}}$ & $\overline{82.3}$ & $\overline{\overline{76.4}}$ & $\overline{\overline{64.5}}$ \\
\hline HaellI_5 || TIC_r & 13.3 & 78.3 & 92.3 & 96.3 & 96.3 & 100 & 100 & $\overline{36.4}$ & $\overline{97.4}$ & $\overline{78.7}$ & $\overline{32.1}$ & $\overline{58.0}$ & $\overline{68.8}$ & $\overline{76.5}$ & $\overline{86.1}$ & $\overline{95.4}$ & $\overline{85.3}$ & $\overline{\overline{79.3}}$ & $\overline{61.8}$ \\
\hline Haelll_6 ||ATM_r & 13.3 & 78.3 & 92.3 & 96.3 & 96.3 & 100 & 100 & 100 & $\overline{33.5}$ & $\overline{81.1}$ & $\overline{31.2}$ & $\overline{55.8}$ & $\overline{66.5}$ & $\overline{74.0}$ & $\overline{83.6}$ & $\overline{92.9}$ & $\overline{87.8}$ & $\overline{81.8}$ & $\overline{59.6}$ \\
\hline HaellI_7 || SXT & 13.3 & 78.3 & 92.3 & 96.3 & 96.3 & 100 & 100 & 100 & 100 & $\overline{4.3}$ & 30.9 & 41.0 & 50.7 & 5 & 65.8 & $\overline{74.3}$ & 86.7 & 81.6 & 45.0 \\
\hline Ssil_1 || C/GM & 12.5 & 75.0 & 88.9 & 92.9 & 92.9 & 96.6 & 96.6 & 96.6 & 96.6 & 96.6 & $\overline{-47.8}$ & $\overline{46.2}$ & $\overline{\overline{42.1}}$ & $\overline{40.7}$ & $\overline{36.6}$ & $\overline{33.8}$ & $\overline{27.2}$ & $\overline{25.3}$ & $\overline{43.3}$ \\
\hline Ssil_2 $\| \mathrm{GM} / \mathrm{C}$ & 12.5 & 75.0 & 88.9 & 92.9 & 92.9 & 96.6 & 96.6 & 96.6 & 96.6 & 96.6 & 100 & $\overline{14.1}$ & $\overline{86.5}$ & $\overline{78.8}$ & $\overline{70.1}$ & $\overline{62.0}$ & $\overline{47.0}$ & $\overline{42.8}$ & $\overline{94.1}$ \\
\hline Ssil_3 \| AN_r & 13.3 & 69.6 & 84.6 & 88.9 & 88.9 & 92.9 & 92.9 & 92.9 & 92.9 & 92.9 & 96.6 & 96.6 & $\overline{41.3}$ & $\overline{86.2}$ & $\overline{81.9}$ & $\overline{73.0}$ & $\overline{56.1}$ & $\overline{51.2}$ & $\overline{92.1}$ \\
\hline Ssil_4 || NET_s & 0.0 & 94.1 & 70.0 & 76.2 & 66.7 & 72.7 & 72.7 & 72.7 & 72.7 & 72.7 & 69.6 & 69.6 & 63.6 & $\overline{49.8}$ & 89.8 & 81.1 & 63.7 & $\underline{58.6}$ & 78.5 \\
\hline Ssil_5 || NN_s & 18.2 & 73.7 & 90.9 & 78.3 & 78.3 & 83.3 & 83.3 & 83.3 & 83.3 & 83.3 & 80.0 & 80.0 & 75.0 & 66.7 & $\overline{58.2}$ & 90.6 & 72.0 & $\overline{66.4}$ & 74.3 \\
\hline Ssil_6 || CIP_S & 15.4 & 66.7 & 83.3 & 88.0 & 96.0 & 92.3 & 92.3 & 92.3 & 92.3 & 92.3 & 88.9 & 88.9 & 84.6 & 60.0 & 72.7 & $\overline{58.0}$ & $\overline{80.9}$ & $\overline{75.0}$ & $\overline{65.8}$ \\
\hline Ssil_7 || TIC_s & 18.2 & 73.7 & 72.7 & 78.3 & 78.3 & 83.3 & 83.3 & 83.3 & 83.3 & 83.3 & 80.0 & 80.0 & 75.0 & 66.7 & 80.0 & \begin{tabular}{|l|}
72.7 \\
\end{tabular} & 56.6 & $\underline{93.8}$ & $\underline{49.9}$ \\
\hline Ssil_8\|AN_s & 14.3 & 63.6 & 80.0 & 84.6 & 84.6 & 88.9 & 88.9 & 88.9 & 88.9 & 88.9 & 92.9 & 92.9 & 96.3 & 57.1 & 69.6 & 80.0 & 69.6 & $\overline{37.8}$ & $\overline{45.4}$ \\
\hline emrA_hm \|ATM_s & 14.3 & 72.7 & 88.0 & 92.3 & 92.3 & 96.3 & 96.3 & 96.3 & 96.3 & 96.3 & 92.9 & 92.9 & 88.9 & 66.7 & 78.3 & 96.0 & 78.3 & 84.6 & 50.3 \\
\hline
\end{tabular}



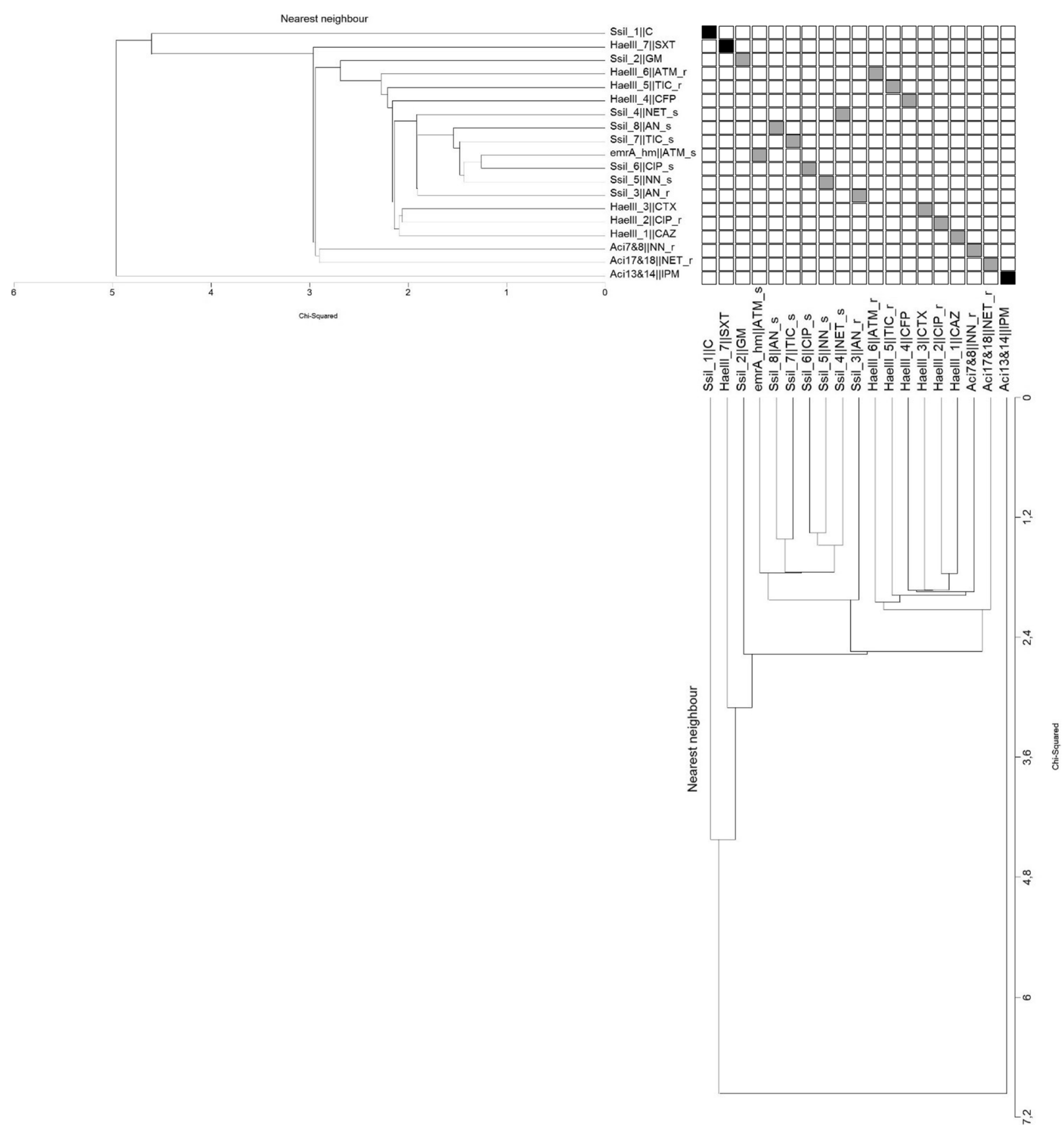

Fig. 1 Phylogenetic trees for different pairs of genetic polymorphisms and resistance/susceptibility features. Branches order obtained based on nearest neighbor method and length-distance calculation based on

with resistance to chloramphenicol and gentamycin; (2) 45 and 55 bp DNA insertions in the same locus, identified using the HaeIII enzyme, combined with trimethoprim/ sulfamethoxazole resistance or susceptibility patterns; and (3) 184 or 1374 bps DNA length polymorphisms in the second genomic region (see tree new PCR region, Table 4), $\chi^{2}$ method. Significant $(p<0.05)$ differences indicated in black boxes were identified based on cut-off $\chi^{2}$ value $=4$

identified in our study for genotypes 1 to 6 and 7 to 16 , in combination with imipenem resistance, characteristic for pattern I or susceptibility features, characteristic for other patterns (Table 6).

The presence of $A$. baumannii genotypes over a period of 4 years in the hospital wards (Table 7), and location of 
Table 7 Presence of A. baumannii genotypes over a period of 4 years

\begin{tabular}{|c|c|c|}
\hline $\begin{array}{l}\text { Year of isolation of the strain } \\
\text { (number of genotypes determined) }\end{array}$ & Ward & $\begin{array}{l}\text { Genotype } \\
\text { (number of isolates) }\end{array}$ \\
\hline \multirow[t]{5}{*}{$2006_{(5)}$} & ICU & $8(3), 10,9,15$ \\
\hline & NS & 8 (3), 7 (2) \\
\hline & $\mathrm{R}$ & $8(2)$ \\
\hline & $\mathrm{E}$ & 7 \\
\hline & $\mathrm{G}$ & 10 \\
\hline \multirow[t]{9}{*}{$2005_{(6)}$} & ICU & $1,8(2), 15,10$ \\
\hline & $\mathrm{R}$ & $8,10,13$ \\
\hline & PS & 8 \\
\hline & NS & 10 \\
\hline & $\mathrm{O}$ & $4(2)$ \\
\hline & G\&ES & 15 \\
\hline & $\mathrm{SC}$ & 15 \\
\hline & Nef & 8 \\
\hline & G\&VS & 10 \\
\hline \multirow[t]{8}{*}{$2004_{(7)}$} & ICU & 5 \\
\hline & $\mathrm{O}$ & 15 \\
\hline & $\mathrm{OC}$ & 4,2 \\
\hline & $\mathrm{E}$ & 15 \\
\hline & $\mathrm{R}$ & 15,3 \\
\hline & NS & 11,15 \\
\hline & G & 4 \\
\hline & $\mathrm{N}$ & 16 \\
\hline \multirow[t]{5}{*}{$2003_{(3)}$} & ICU & $6(2), 12$ \\
\hline & G\&ES & $6(2)$ \\
\hline & $\mathrm{O}$ & $6(2)$ \\
\hline & $\mathrm{N}$ & 6 \\
\hline & $\mathrm{D}$ & 14 \\
\hline
\end{tabular}

Abbreviations for wards: D-Dermatology, E-Endocrinology, GGeriatrics, G\&ES - General and Endocrine Surgery, G\&VS-General and Vascular Surgery, ICU-Intensive Care Unit, N-Neurology, Nef-Nephrology, NS-Neurosurgery, O-Orthopedic, OC-Orthopedic Outpatient Clinic, PS-Plastic Surgery, R-Rehabilitation, SC—Surgical Outpatient Clinic

determined genotypes over a 4-year period in hospital wards (Table 8), was also assessed. This analysis provides a possibility to identify the presence of specific isolates in various wards over the period of several years. We suggest that such analyses may be useful in epidemiological studies on the origin and migration of particular bacterial strains between different wards of an investigated hospital. It also gives the possibility to analyze the strains regardless of the time period in which they were collected.

\section{Conclusions}

In conclusion, 16 different genotypes out of 51 MDR $A$. baumannii clinical isolates were identified in our study. Based on combined comparative analysis of genetic and
Table 8 Location of determined genotypes over a 4-year period in hospital wards

\begin{tabular}{lll}
\hline Genotype & Year $_{(\text {number of genotypes) }}$ & Hospital ward(s) \\
\hline 15 & $2006_{(1)}$ & ICU \\
& $2005_{(3)}$ & ICU, G\&ES, SC \\
& $2004_{(4)}$ & O, E, R, NS \\
8 & $2006_{(8)}$ & ICU, NS, R \\
& $2005_{(5)}$ & ICU, R, Nef \\
10 & $2006_{(2)}$ & ICU, G \\
& $2005_{(4)}$ & ICU, R, NS, G\&VS \\
4 & $2005_{(2)}$ & O \\
& $2004_{(2)}$ & OC, G \\
6 & $2003_{(7)}$ & ICU, G\&ES, O, N \\
7 & $2006_{(3)}$ & E \\
1 & $2005_{(1)}$ & ICU \\
2 & $2004_{(1)}$ & ICU \\
3 & $2004_{(1)}$ & R \\
5 & $2004_{(1)}$ & ICU \\
9 & $2006_{(1)}$ & ICU \\
11 & $2004_{(1)}$ & NS \\
12 & $2003_{(1)}$ & ICU \\
13 & $2005_{(1)}$ & R \\
14 & $2003_{(1)}$ & D \\
16 & $2004_{(1)}$ & N \\
\hline
\end{tabular}

Abbreviations for wards: D-Dermatology, E-Endocrinology, GGeriatrics, G\&ES-General and Endocrine Surgery, G\&VS-General and Vascular Surgery, ICU-Intensive Care Unit, N-Neurology, Nef-Nephrology, NS-Neurosurgery, O-Orthopedic, OC-Orthopedic Outpatient Clinic, PS-Plastic Surgery, R-Rehabilitation, SC-Surgical Outpatient Clinic

resistance patterns, two significantly different patterns of DNA polymorphisms in the CRISPR coding region, resistance to chloramphenicol and gentamycin features, and resistance or susceptibility to trimethoprim/sulfamethoxazole, specific groups of isolates were identified. Out of 19 genetic markers and antibiotic resistance features, three of them were shown to be statistically significantly different using two statistical tools (Table 6, Fig. 1). In addition, 184 or 1374 bp DNA length polymorphisms in genomic region no. 2, located upstream of the GTP cyclohydrolase I gene, with the Locus_tag = "ABTJ_01152", associated in $94 \%$ with susceptibility to imipenem, was identified. Finally, the highest genetic diversity, determined within the DNA polymerase III subunit gamma/tau gene, can be recommended for future genotyping of multidrug-resistant A. baumannii strains. We suggest that the optimized methods, proposed in this report and based on combination of Repeated Sequences and Whole Genome Alignment Differential Analysis (RS\&WGADA), can be useful in epidemiological studies concerning specific strains of pathogenic bacteria present in investigated hospitals. 
Authors' contributions R.K.: data curation, investigation, writingoriginal draft, writing - editing and review, supervision. A.N-Z.: data curation, investigation, writing — original draft and review. G.W.: writing-editing and review.

Funding This work was partly supported by the State Committee for Scientific Research, grant no. KBN 2P05D 10128.

Data availability Not applicable.

Code availability Not applicable.

\section{Declarations}

Ethics approval The Bioethics Committee waived the need for consent from all patients from whom bacterial strains were isolated and used in this study. Decision no. KB 248/2016 was issued by the Local Bioethics Committee at the Nicolaus Copernicus University Ludwik Rydygier Collegium Medicum in Bydgoszcz, Poland.

Consent to participate Not applicable.

Consent for publication Not applicable.

Conflicts of interest The authors declare that they have no conflict of interest.

Open Access This article is licensed under a Creative Commons Attribution 4.0 International License, which permits use, sharing, adaptation, distribution and reproduction in any medium or format, as long as you give appropriate credit to the original author(s) and the source, provide a link to the Creative Commons licence, and indicate if changes were made. The images or other third party material in this article are included in the article's Creative Commons licence, unless indicated otherwise in a credit line to the material. If material is not included in the article's Creative Commons licence and your intended use is not permitted by statutory regulation or exceeds the permitted use, you will need to obtain permission directly from the copyright holder. To view a copy of this licence, visit http://creativecommons.org/licenses/by/4.0/.

\section{References}

Adams MD, Goglin K, Molyneaux N, Hujer KM, Lavender H, Jamison JJ, MacDonald IJ, Martin KM, Russo T, Campagnari AA, Hujer AM, Bonomo RA, Gill SR (2008) Comparative genome sequence analysis of multidrug-resistant Acinetobacter baumannii. J Bacteriol 190:8053-8064. https://doi.org/10.1128/JB.00834-08

Ahmed SS, Alp E (2015) Genotyping methods for monitoring the epidemic evolution of Acinetobacter baumannii strains. J Infect Dev Ctries 9:347-354. https://doi.org/10.3855/jidc.6201

Antunes LC, Visca P, Towner KJ (2014) Acinetobacter baumannii: evolution of a global pathogen. Pathogens and Disease 71:292301. https://doi.org/10.1111/2049-632X.12125

Barbe V, Vallenet D, Fonknechten N, Kreimeyer A, Oztas S, Labarre L, Cruveiller S, Robert C, Duprat S, Wincker P, Ornston LN, Weissenbach J, Marlière P, Cohen GN, Médigue C (2004) Unique features revealed by the genome sequence of Acinetobacter sp. ADP1, a versatile and naturally transformation competent bacterium. Nucleic Acids Res 32:5766-5779. https://doi.org/10.1093/ nar/gkh910
Ergin A, Hascelik G, Eser OK (2013) Molecular characterization of oxacillinases and genotyping of invasive Acinetobacter baumannii isolates using repetitive extragenic palindromic sequence-based polymerase chain reaction in Ankara between 2004 and 2010. Scand J Infect Dis 45:26-31. https://doi.org/10.3109/00365548. 2012.708782

Irfan S, Turton JF, Mehraj J, Siddiqui SZ, Haider S, Zafar A, Memon B, Afzal O, Hasan R (2011) Molecular and epidemiological characterisation of clinical isolates of carbapenem-resistant Acinetobacter baumannii from public and private sector intensive care units in Karachi, Pakistan. J Hosp Infect 78:143-148. https://doi. org/10.1016/j.jhin.2011.01.029

Karah N, Samuelsen Ø, Zarrilli R, Sahl JW, Wai SN, Uhlin BE (2015) CRISPR-cas Subtype I-Fb in Acinetobacter baumannii: evolution and utilization for strain subtyping. PLoS ONE 10(2):e0118205. https://doi.org/10.1371/journal.pone.0118205

Katoh K, Misawa K, Kuma K, Miyata T (2002) MAFFT: a novel method for rapid multiple sequence alignment based on fast Fourier transform. Nucleic Acids Res 15:3059-3066

Martín-Lozano D, Cisneros JM, Becerril B, Cuberos L, Prados T, Ortíz-Leyba C, Cañas E, Jachón P (2002) Comparison of a repetitive extragenic palindromic sequence-based PCR method and clinical and microbiological methods for determining strain sources in cases of nosocomial Acinetobacter baumannii bacteremia. J Clin Microbiol 40:4571-4575

Minandri F, D’Arezzo S, Antunes LCS, Pourcel C, Principe L, Petrosillo N, Visca P (2012) Evidence of diversity among epidemiologically related carbapenemase-producing Acinetobacter baumannii strains belonging to international clonal lineage II. J Clin Microbiol 50:590-597. https://doi.org/10.1128/JCM.05555-11

Nabil K, Samuelsen Ø, Zarrilli R, Sahl JW, Wai SN, Uhlin BE (2015) CRISPR-cas subtype I-Fb in Acinetobacter baumannii: evolution and utilization for strain subtyping. PLoS ONE 10(2):e0118205. https://doi.org/10.1371/journal.pone.0118205

Nowak-Zaleska A, Krawczyk B, Kotłowski R, Mikucka A, Gospodarek E (2008) Amplification of a single-locus variable-number direct repeats with restriction fragment length polymorphism (DR-PCR/ RFLP) for genetic typing of Acinetobacter baumannii strains. Pol J Microbiol 57:11-17

Nowak-Zaleska A, Wieczór M, Czub J, Nierzwicki Ł, Kotłowski R, Mikucka A, Gospodarek E (2016) Correlation between the number of Pro-Ala repeats in the EmrA homologue of Acinetobacter baumannii and resistance to netilmicin, tobramycin, imipenem and ceftazidime. J Glob Antimicrob Resist 7:145-149. https:// doi.org/10.1016/j.jgar.2016.09.004

Pourcel C, Minandri F, Hauck Y, D’Arezzo S, Imperi F, Vergnaud G, Visca P (2011) Identification of variable-number tandem-repeat (VNTR) sequences in Acinetobacter baumannii and interlaboratory validation of an optimized multiple-locus VNTR analysis typing scheme. J Clin Microbiol 49:539-548. https://doi.org/10. 1128/JCM.02003-10

Sambrook J, Fritsch EF, Maniatis T (1989) Molecular cloning: a laboratory manual, 2nd edn. Cold Spring Harbor Laboratory Press, Cold Spring Harbor

Shariat N, Dudley EG (2014) CRISPRs: molecular signatures used for pathogen subtyping. Appl Environ Microbiol 80:430-439. https:// doi.org/10.1128/AEM.02790-13

Skariyachan S, Taskeen N, Ganta M, Krishna BV (2019) Recent perspectives on the virulent factors and treatment options for multidrug-resistant Acinetobacter baumannii. Crit Rev Microbiol 45:315-333. https://doi.org/10.1080/1040841X.2019.1600472

Smith MG, Gianoulis TA, Pukatzki S, Mekalanos JJ, Ornston LN, Gerstein M, Snyder M (2007) New insights into Acinetobacter baumannii pathogenesis revealed by high-density pyrosequencing and transposon mutagenesis. Genes Dev 21:601-614. https://doi. org/10.1101/gad.1510307 
Touchon M, Cury J, Yoon E-J, Krizova L, Cerqueira GC, Murphy C, Feldgarden M, Wortman J, Clermont D, Lambert T, GrillotCourvalin C, Nemec A, Courvalin P, Rocha EPC (2014) The genomic diversification of the whole Acinetobacter genus: origins, mechanisms, and consequences. Genome Biol Evol 6:2866-2882. https://doi.org/10.1093/gbe/evu225

Turton JF, Matos J, Kaufmann ME, Pitt TL (2009) Variable number tandem repeat loci providing discrimination within widespread genotypes of Acinetobacter baumannii. Eur J Clin Microbiol Infect Dis 28:499-507. https://doi.org/10.1007/s10096-008-0659-3

Villalón P, Valdezate S, Cabezas T, Ortega M, Garrido N, Vindel A, Medina-Pascual MJ, Saez-Nieto JA (2015) Endemic and epidemic Acinetobacter baumannii clones: a twelve-year study in a tertiary care hospital. BMC Microbiol 15:47. https://doi.org/10.1186/ s12866-015-0383-y
Wong D, Nielsen TB, Bonomo RA, Pantapalangkoor P, Luna B, Spellberg B (2017) Clinical and pathophysiological overview of Acinetobacter infections: a century of challenges. Clin Microbiol Rev 30:409-447. https://doi.org/10.1128/CMR.00058-16

Zhou K, Aertsen A, Michiels CW (2014) The role of variable DNA tandem repeats in bacterial adaptation. FEMS Microbiol Rev 38:119-141. https://doi.org/10.1111/1574-6976.12036

Publisher's note Springer Nature remains neutral with regard to jurisdictional claims in published maps and institutional affiliations. 